\title{
NEW GREEN ENERGY: OCEAN WAVE ENERGY CONVERSION PHYSICAL TURBINE MODELS AND DESIGNS PHOTOS PRESENTATION AND EXPLANATIONS FOR LARGE SCALE MANUFACTURING
}

\section{S.S. ARULAPPAN}

Associate Professor, Department of Mechanical Engineering, National Institute of Technology,

Tiruchirappalli, Tamil Nadu, India

Energy needs are large and inevitable but energy production by Thermal and Nuclear power producing plants may have to be gradually withdrawn as they are directly and indirectly harmful and dangerous to the entire world where all human beings and all other living things will have to live peacefully.

The better alternative energy like wind energy is already being exploited and used even though by some less effective wind turbines [1] which can be improved using higher efficiency wind turbines as per the new design of the blades and experimental results mentioned in the two international award winning papers which are published internationally [2] by the same author of this technical research paper.

One more Mega Energy Source which is much more powerful is waiting for millions of years and which can last for so many more years has to be immediately used to avoid further pollution by existing thermal power plants and the dangerous Nuclear plants.

The Energy source the technocrats may opt now is Ocean Wave Energy. Wave Energy is a Green energy and it has much more energy supplying capacity than other sources of energy. Water is a Denser Medium and Mighty Ocean waves can Push the blades of the Turbine much more powerfully than the diffused wind energy and harmful thermal and Nuclear energy.

KEYWORDS: Ocean Wave Energy, Physical Turbine Models, Designs, Mighty Green Energy Source, Reciprocating and Rotary Turbine Models, Commercial Large Scale Manufacturing \& Purchase From the Author
\end{abstract}

Received: Jul 24, 2017; Accepted: Aug 08, 2017; Published: Aug 16, 2017; Paper Id.: IJMPERDAUG201742

\section{INTRODUCTION}

Energy production from ocean waves is particularly Non Polluting Green Energy Supplying mega Capability from the Vast Ocean water body. Ocean water which is a denser medium than the wind energy hence these turbines can produce enormous amount of energy for a much smaller turbine from the water waves which are violently moving here and there all the time Day and Night and all around our Earth. This Ocean wave energy is Everlasting Giant source of Energy.

The ocean waves has movements of ups and down and forward and back ward Mighty Movements always all throughout the days with nonstop continuation. Hence Why not these movements can be used to move or rotate the turbines and intern the turbines can be coupled with an alternator to produce electricity. The photographs of 
new physical models are presented.

Reciprocating and Rotating Physical Models are fabricated with conceptual new design ideas and presented in these technical papers. These ideas are actually new to the world which may be called as inventions.

The necessity of these new inventions are for the better and more effective Green energy conversion devices than the already existing other Polluting Thermal Power Plants.

\section{JUSTIFICATION FOR WAVE ENERGY CONVERSION}

This physical model is presented in the following Photograph. It can work by continuous movement from the one behind the other moving ocean waves. If this Turbine is made with larger size and erected the into the Sea water half immersed it start producing the power. The bottom curved blades will be pushed by the moving water waves and the upper curved blades which are above the water level can come down and receive the blow and the pushing force of the waves continuously.

Even though the waves are intermittent and one behind the other the turbine as a whole can continuously work and produce electric power with the help of alternator coupled to it. A larger scale model can be designed and manufactured and erected all throughout the world by commercial manufacturers by buying the conceptual idea and the actual Physical model from the Author of this Technical Paper.

Solar Thermal rays, Solar gravitational attraction towards Earth, Lunar attraction, Earth gravitational attraction, Spinning action of the Earth and Wind forces are the causes for Water waves and Tidal ups and downs of the Ocean

These forces are everlasting as long as Sun, Earth and Moon which is Earth's natural Satellite are there in Physical existence. Hence Ocean waves will have to be there to dissipate the extra energy it receives from those giant sources.

Clearly one can decide to harness this very large everlasting Green energy source of energy without polluting the plant Earth by other harmful source of energy any more. Can this not be done?

\section{ALL NEW OCEAN WAVE ENERGY CONVERSION TURBINES PHYSICAL MODELS}

This model can harness ocean wave motion into reciprocating motion or continuous rotation by the pushing water waves of the ocean waves. The photograph can give a first look of the physical model. This model can be manufactured into a larger size so that higher commercial power production can be done. This turbine may not look like regular hydraulic turbine.

However the ocean waves have large kinetic energy in the osculating push and pull form. Mighty and powerful hydraulic movements are left unutilized so far. The enormous energy can be well utilized for all our energy needs without making any harmful emissions.

Nuclear and Thermal energy power plants can be gradually closed if Ocean wave energy is tapped in large quantum. All around the world Ocean waves are waiting so that thermal plants and nuclear plants can be stopped from further producing harmful and dangerous pollutions.

Ocean wave energy is Not an Alternate Energy but this can become a Main Source of energy and that too everlasting. Ocean wave energy is a Non Polluting Ever Lasting GREEN ENERGY. As long as Solar Radiation is received by Earth Ocean will be having the Wave Energy. 

Presentation and Explanations for Large Scale Manufacturing

Waves are there Throughout the Year throughout the Day so this Energy is Reliable and it is Definitely Worth investing Money into this Giant Source of Energy. Can it not be done?

\section{MODEL 1}

Straight Bladed Turbine half immersed can convert both forward and backward with the frame kept horizontal to the motion of the waves as reciprocating motions by Immersing Bottom Half of this linearly moving turbine.

This motion can be utilised to produce electricity by converting it into rotary motion and coupling an alternator to take out electrical energy as the output from the alternator.

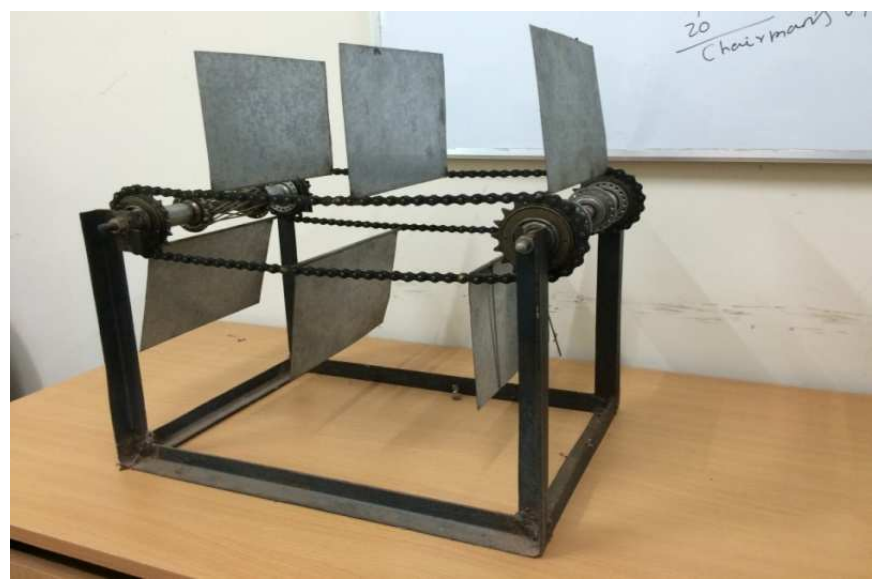

Figure 1

\section{MODEL 2}

One sided Curved Vanes of the Turbine bottom half alone can be immersed into ocean water so that it can convert ocean wave movement into a continuous motion by receiving one after the other waves getting generated naturally in the Ocean. The waves can push one after the other the Turbine Vanes / Blades. This movement can be used to rotate an Alternator to produce Electricity.

All our need for electrical energy can be fulfilled by the Mighty and Vast Ocean. No need to worry about Energy Requirements. This Energy is available free of cost and free from all types of pollutions.

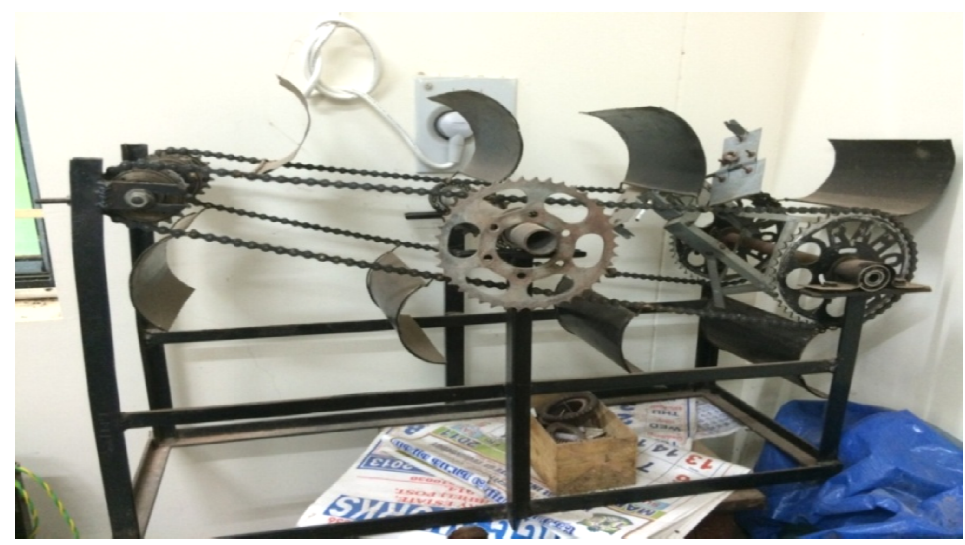

Figure 2 


\section{MODEL 3}

Forward and Backward movements of the ocean water can be converted into continuous rotary motion by the Model 3 Turbine shown in the following Photograph. This turbine is like usual hydraulic turbine but immersed into ocean water waves completely. The movements of the water waves can push the blades and rotate the blades only in one direction for a particular angle of inclination of the blades and can rotate continuously for both the pushing and returning action of the waves.

Finally the Kinetic Energy of the waves can become rotary motion of the turbine and this turbine can be coupled to an alternator to produce electricity.

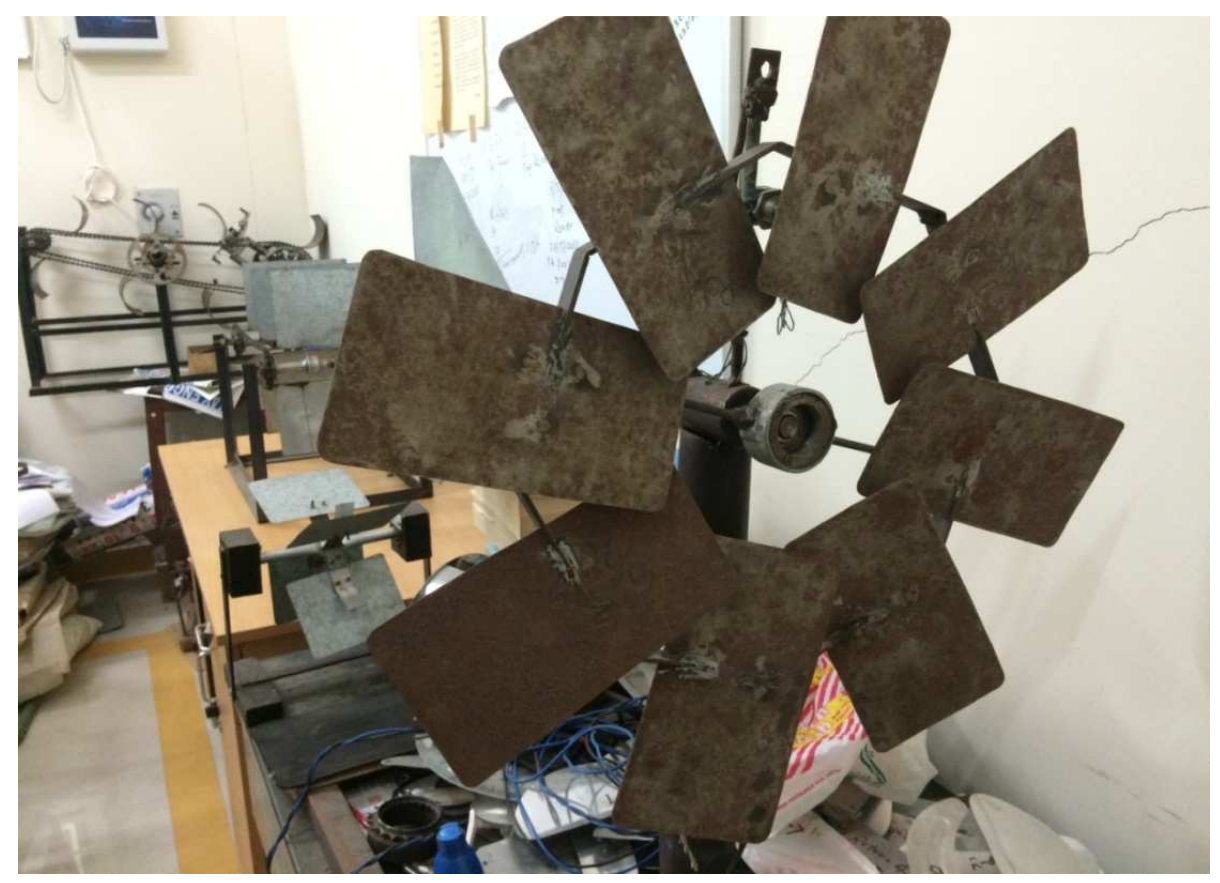

Figure 3

\section{CONCLUSIONS}

Ocean wave energy is much more superior and cheaper than the Wind Energy and other Thermal and Nuclear Power plants both by overall Cost and Power produced because water is a denser medium than air and Direct Mechanical Kinetic Energy is available in large quantum that too free of Cost and also everlasting. Large water wave turbines can produce much higher energy from the denser medium of Water than the Wind Turbines which are working with much Diffused medium of Air. Hence one can immediately and confidently invest money into this research and buy the Basic Designs and Physical models from the Author of this research Paper.

\section{REFERENCES}

1. S.S. Arulappan, Numerical, Experimental and Analytical Investigation for Higher Wind Turbine Efficiency, Published in International Journal of Mechanical and Production Engineering Research and Development, TJPRC in August 2015. This paper received International Best Research Paper Award in October 2015.

2. S.S. Arulappan, 'Experimental and CFD Investigations Analogy for Wind Turbine Efficiency Using Commercial Small Fan 
New Green Energy: Ocean Wave Energy Conversion Physical Turbine Models and Designs Photos

\section{Presentation and Explanations for Large Scale Manufacturing}

Blades' Published in International Journal of Mechanical and Production Engineering Research and Development, TJPRC in October 2015. This paper Received the International Best Research Paper Award in January 2016. 
